\title{
Prefrontal cortex: Role in acquisition of overlapping associations and transitive inference
}

\author{
Loren M. DeVito, ${ }^{1}$ Christine Lykken, ${ }^{1}$ Benjamin R. Kanter, and Howard Eichenbaum ${ }^{2}$
}

Center for Memory and Brain, Boston University, Boston, Massachusetts 02215, USA

\begin{abstract}
"Transitive inference" refers to the ability to judge from memory the relationships between indirectly related items that compose a hierarchically organized series, and this capacity is considered a fundamental feature of relational memory. Here we explored the role of the prefrontal cortex in transitive inference by examining the performance of mice with selective damage to the medial prefrontal cortex. Damage to the infralimbic and prelimbic regions resulted in significant impairment in the acquisition of a series of overlapping odor discrimination problems, such that animals with prefrontal lesions required twice as many trials to learn compared to sham-operated controls. Following eventually successful acquisition, animals with medial prefrontal lesions were severely impaired on a transitive inference probe test, whereas they performed as well as controls on a test that involved a nontransitive judgment from a novel odor pairing. These results suggest that the prefrontal cortex is part of an integral hippocampal-cortical network essential for relational memory organization.
\end{abstract}

Although relatively smaller and less developed than in primates, the infralimbic and prelimbic regions of the rodent medial prefrontal cortex are thought to be analogous to primate dorsolateral prefrontal cortex due to the projections originating from the mediodorsal nucleus of the thalamus (Verwer et al. 1997; Ongur and Price 2000). In addition, the collection of behaviors described as "executive functions," such as attentional selection, behavioral inhibition, task switching, planning, and decision-making, that have been attributed to primate dorsolateral prefrontal cortex are also supported by the medial prefrontal cortex in the rodent (Brown and Bowman 2002; Dalley et al. 2004; Rich and Shapiro 2009). It has further been suggested that both the primate dorsolateral prefrontal cortex and rodent medial prefrontal cortex also contribute importantly to the organization and integration of information that supports episodic memory (Carpenter et al. 2000; Simons and Spiers 2003; Farovik et al. 2008); however, the precise role of the prefrontal cortex in relational memory is not clear.

"Episodic memory" refers to the encoding and retrieval of specific features about past experiences, such as the specific places and items encountered at particular times. An important aspect of episodic memory is the ability to use information from past events to guide behavior flexibly in novel situations in which only some cues may be available for retrieval. It has been suggested that this capacity is supported by the acquisition of a relational network that links memories sharing common elements and the ability to subsequently retrieve the entire network when prompted by a subset of the elements (Eichenbaum 2004). Transitive inference is a relational memory task that relies on the ability to integrate experiences sharing overlapping features and to use that information to guide judgments about those indirectly related experiences. Rats and monkeys with damage to the fornix, hippocampus, and perirhinal and entorhinal cortices can learn the items associated with a memory, but are impaired when asked to make judgments about those indirectly related items (Bunsey and Eichenbaum

\footnotetext{
1These authors contributed equally to this work.

${ }^{2}$ Corresponding author.

E-mail hbe@bu.edu; fax (617) 353-1414.

Article is online at http://www.learnmem.org/cgi/doi/10.1101//m.1685710.
}

1996; Dusek and Eichenbaum 1997; Buckmaster et al. 2004; Van der Jeugd et al. 2009; DeVito et al. 2010). In humans, the hippocampus is activated when subjects perform transitive inference judgments (Heckers et al. 2004; Zalesak and Heckers 2009).

Because the hippocampus and prefrontal cortex are connected by strong anatomical projections (Laroche et al. 2000; Thierry et al. 2000), it has been suggested that the prefrontal cortex might interact with the hippocampus in support of episodic memory (Buckner et al. 1999; Smith and Jonides 1999; Petrides 2000). Functional imaging studies in humans have suggested that the prefrontal cortex is engaged during transitive inference (Goel and Dolan 2001; Acuna et al. 2002; Kroger et al. 2002), and patients with damage to the prefrontal cortex demonstrate an impaired ability for relational reasoning (Waltz et al. 1999), suggesting that transitive inference provides a useful paradigm for examining prefrontal-hippocampal interactions in relational memory.

Although, as described above, many studies have indicated that the hippocampus and surrounding cortical structures play an important role in transitive inference and relational memory in animals, the contribution of the prefrontal cortex in animals is unknown. Here we investigated the role of the medial prefrontal cortex in transitive inference in the mouse. Following procedures previously used to examine hippocampal function, we initially trained animals with prefrontal lesions and sham-operated controls on a series of overlapping odor discrimination problems (A+ vs. B - , B + vs. C - , C + vs. D- , D+ vs. E - ; where each letter indicates a different odor). These discriminations were progressively intermixed as multiple problems across four stages of training, and then, during continued testing on all the training problems, the capacity for transitive inference was assessed by presenting the animals with occasional choices between indirectly related items B and D, as well as on a control pair (A vs. E) that did not require a transitive judgment (see Fig. 1).

\section{Results}

\section{Histology}

Ibotenic acid infusions resulted in a substantial loss of cells within the medial wall of the prefrontal cortex, including the prelimbic 
A

\begin{tabular}{|l|l|}
\hline Stage & Training Schedule \\
\hline I & $\begin{array}{l}\text { Day One: } \\
\text { Session 1: AB (8 trials) } \\
\text { Session 2: BC (8 trials) } \\
\text { Day Two: } \\
\text { Session 1: CD (8 trials) } \\
\text { Session 2: DE (8 trials) }\end{array}$ \\
\hline II & $\begin{array}{l}\text { Session 1: AB \& BC (4 trials each) } \\
\text { Session 2: CD \& DE (4 trials each) }\end{array}$ \\
\hline III & $\begin{array}{l}\text { Session 1: AB, BC, CD \& DE (2 trials each) } \\
\text { Session 2: Repeat }\end{array}$ \\
\hline$N$ & $\begin{array}{l}\text { Session 1: Pseudorandom presentation of } \\
\text { all pairs; 2 trials of each pair } \\
\text { Session 2: Repeat }\end{array}$ \\
\hline
\end{tabular}

B

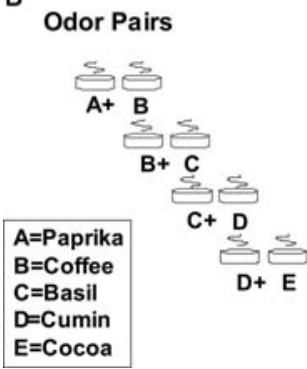

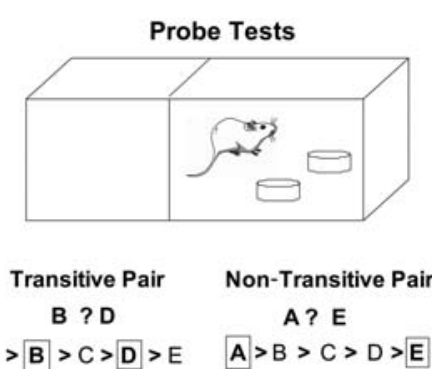

$A>B>C>D>E \quad A>B>C>D>E$

Figure 1. Transitive inference training and testing protocol. (A) Description of the stages to train animals on each of the odor pairs. (B) Schematic of the behavioral apparatus used to train and test the animals. Each letter refers to a specific odor used in the task. The " +" indicates the rewarded odor in each pairing.

and infralimbic regions and dorsal cingulate gyrus (Fig. 2A,B). An average of $43 \%$ of the medial prefrontal cortex was damaged across all animals, ranging from $12 \%$ to $81 \%$ (Fig. 2C). Two animals had additional damage to the medial orbital cortex, and two animals had unilateral damage to the ventral and lateral orbital cortices. The extent of damage in the prefrontal cortex was not correlated with acquisition of the odor pairs $\left(r_{(10)}=\right.$ $0.20, P=0.576$ ) or performance on the $\mathrm{BD}$ (transitive) probe test $\left(r_{(10)}=-0.27, P=0.446\right)$.

\section{Acquisition of odor pairs}

During Stage 1 , the animals are presented with a block of A+vs. $\mathrm{B}-$ discrimination trials in the first session and a block of $\mathrm{B}+$ vs. C- discrimination trials in the second session on the first day (Fig. 1). The following day, they are presented with the $\mathrm{C}+$ vs. $\mathrm{D}-$ and $\mathrm{D}+$ vs. $\mathrm{E}-$ discriminations in the same manner. During Stages 2 and 3, the number of trials within each block is progressively reduced, such that all discriminations were presented in $1 \mathrm{~d}$ across the two training sessions; however, in Stage 4, all four discriminations were intermixed and presented together in a pseudorandom fashion.

Both groups of animals successfully learned the odor pairs across the four stages of training; however, animals with damage to the prefrontal cortex were significantly impaired in acquisition compared to sham-operated animals. Whereas sham-operated mice completed the training in an average of $16.78 \pm 1.73$ (SEM) d, mice with prefrontal lesions required an average of $30.7 \pm$

$5.33 \mathrm{~d}$ to complete training $\left(t_{(17)}=5.57, P=0.03\right.$; Fig. $\left.3 \mathrm{~A}\right)$. There was a significant group effect across the four stages of training (Two-way ANOVA: group, $F_{(1,75)}=8.87, P=0.004$; stage, $F_{(3,75)}=0.45, P=0.713$; group $\times$ stage interaction, $F_{(3,75)}=1.27$, $P=0.289$; Fig. 3B). Post-hoc analyses ( $t$-tests) were performed to determine if the group difference was significant at each of the four stages. These tests revealed that the prefrontal lesion animals required a significantly greater number of days to reach criterion specifically on Stage $4\left(t_{(17)}=5.07, P=0.038\right)$, the only stage in which the odor pairs were presented in a pseudorandom order.

Despite this impairment in acquisition, both groups of animals performed comparably across the trained odor pairs on the days in which the probe tests were administered (Two-way ANOVA: group, $F_{(1,75)}=0.25, P=0.617$; odor pairs, $F_{(3,75)}=$ $0.93, P=0.431 ;$ group $\times$ pair interaction, $F_{(3,75)}=3.22, P=$ 0.028; Fig. 3C). Post-hoc analyses (t-tests) revealed a trend toward significance on the $\mathrm{BC}$ and $\mathrm{CD}$ pairs, where the prefrontal lesion group performed at higher levels on the $\mathrm{BC}$ pair as compared to sham-operated animals $\left(t_{(17)}=4.07, P=0.06\right)$, and the sham-operated group performed at higher levels on the CD pair as compared to prefrontal lesion animals $\left(t_{(17)}=3.96, P=\right.$ 0.063); despite these trends in the data, there was no significant difference in performance between the two groups on any of the particular odor pairs.

Within the sham-operated group, performance differed significantly across pair types (One-way ANOVA: $F_{(3,35)}=3.72, P=$ $0.021)$, such that performance on the BC pair was significantly lower than performance on all other pairs (AB vs. BC: $P=0.034$; $\mathrm{BC}$ vs. $\mathrm{CD}: P=0.003$; $\mathrm{BC}$ vs. $\mathrm{DE}: P=0.034)$; however, within the lesion group, there was no difference across pair types (One-way ANOVA: $F_{(3,39)}=0.37, P=0.769$ ). These differences in the pattern of performance across the pair types within each group suggest that the prefrontal lesion group was using a different strategy to represent the full set of odor pairs.

\section{Performance on BD (transitive) and AE (nontransitive) probe tests}

Transitive inference was measured by an index of preference between non-end items that were not adjacent in the hierarchy, specifically an item higher in the hierarchy (B) versus an item lower in the hierarchy (D; see Materials and Methods and Fig. 1B). Animals with prefrontal damage were severely impaired compared to sham-operated animals on the transitive BD probe test $\left(t_{(17)}=7.09, P=0.016\right.$; Fig. $\left.4 \mathrm{~A}\right)$. Furthermore, whereas sham-operated animals performed at levels that were significantly above chance $\left(t_{(16)}=36.86, P<0.001\right)$, animals with prefrontal lesions did not $\left(t_{(17)}=0.25, P=0.623\right)$.

As a control for performance with a novel pairing that did not require inferential judgment, we also evaluated preference for the always-rewarded item A over the never-rewarded item $\mathrm{E}$ (Fig. 1B). Performance on the nontransitive AE probe test did not differ significantly between the two groups $\left(t_{(17)}=3.17, P=\right.$ 0.093; Fig. 4B). Both groups performed at levels that were significantly better than chance (sham-operated animals: $t_{(16)}=793.10$, $P<0.001$; prefrontal lesion animals: $\left.t_{(17)}=76.32, P<0.001\right)$. In addition, the two groups spent an equivalent amount of time digging in the probe cups, indicating similar levels of activity and motivation $\left(t_{(306)}=2.71, P=0.101\right)$.

\section{Discussion}

Although previous studies have indicated that the hippocampus and surrounding cortical areas play an important role in transitive 
A
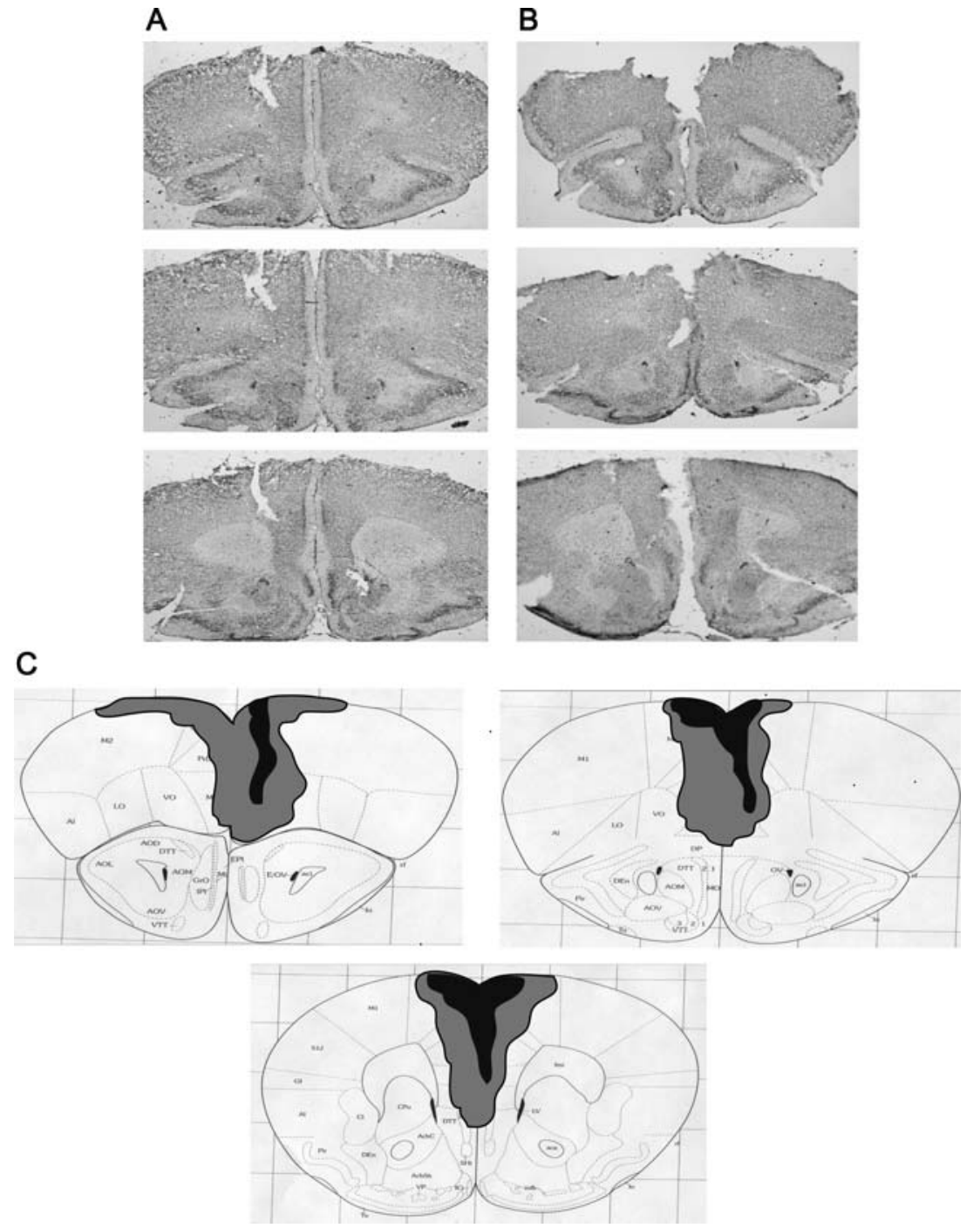

C

Figure 2. Histological verification of the extent of medial prefrontal cortex damage. $(A)$ Representative sections from three levels along the anterior-posterior axis of the mouse prefrontal cortex in a sham-operated animal. $(B)$ Corresponding sections from an animal given ibotenic acid infusions into the medial prefrontal cortex. (C) A diagram shows the extent of the largest (light gray) and smallest (black) lesion across the 10 animals.

inference in rodents, the contribution of the prefrontal cortex has not previously been explored. Here, we demonstrate for the first time that damage to the medial prefrontal cortex produces impairment in the acquisition of odor pairs and a severe deficit in subsequent transitive inference judgments in mice. These results suggest that the prefrontal cortex plays an essential role in the relational memory abilities supported by an integral hippocampal-cortical network.

\section{Animals with damage to the medial prefrontal cortex demonstrate impaired acquisition of overlapping odor pairs}

Mice with damage to the prefrontal cortex required a significantly greater number of trials to learn the odor pairs than did the sham-operated mice. Although animals with prefrontal damage required more trials to learn the training pairs, this impairment emerged only after the first stage of training and was significant only at the fourth stage. In the first stage of training, the animals received a single pair of odor discriminations per session (e.g., a choice between A or B in the first session and a choice between $\mathrm{B}$ or $\mathrm{C}$ in the second session), and both groups were able to learn the pairs at comparable levels. However, animals with damage to the medial prefrontal cortex exhibited increasing difficulty across the following three stages, where they were required to solve multiple discrimination problems presented within each session. In the fourth stage, in which all pairs are presented in a pseudorandom order (instead of a block sequence), the animals exhibited the greatest deficit. It is important to note that in this stage the animal cannot solve the problem by referring to the previously rewarded and nonrewarded elements because the same pairings are not presented consecutively; therefore, damage to the prefrontal cortex specifically impaired the ability to solve the problem when they were presented concurrently with an unpredictable sequence of multiple pairings.

Buckmaster et al. (2004) found that monkeys with damage to the entorhinal cortex were capable of learning the discriminations but failed at the critical transitive judgment test. In addition, Dusek and Eichenbaum (1997) found similar results with rats that received combined perirhinal and entorhinal lesions. Although these animals acquired the discriminations at a rate comparable to sham-operated animals, they were severely impaired in their ability to perform the transitive probe test. Additional evidence suggesting that the medial temporal lobe supports transitive inference comes from a study using paired associate learning. This task, similar to transitive inference, involves training animals on two sets of odor pairs that are associated by a common item. The transitivity test requires inferring associations between these two linked pairs, instead of items associated within a hierarchically organized series. Using this paradigm, Bunsey and Eichenbaum (1996) found that damage to the hippocampus impaired the ability to make the associative inference without affecting the rate at which the odor pairs were learned. These findings suggest that the medial temporal lobe supports the creation of associations between items with common elements, whereas the prefrontal cortex supports the acquisition or organization of the pairwise choices within a related series.

The impaired acquisition of odor pairs exhibited by animals with prefrontal damage may reflect their inability to inhibit previously rewarded responses to particular stimulus elements, or to flexibly switch strategies as the response and reward contingencies change across the odor pairings. The primate dorsolateral prefrontal cortex, as well as the medial prefrontal cortex of the rodent, has been shown to play an important role in attention and inhibitory control processes (Rezai et al. 1993; Dalley et al. 


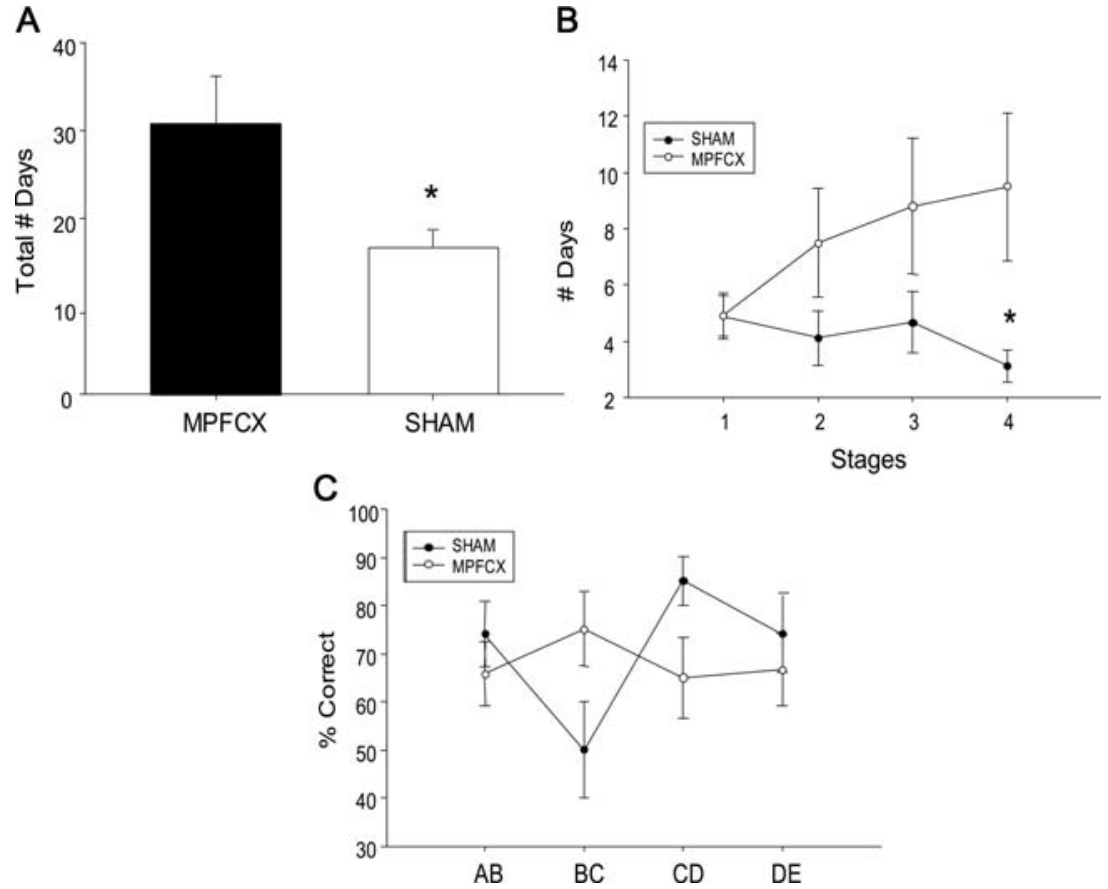

Figure 3. Performance on the odor pairs. $(A)$ Performance $( \pm$ SEM) across all four stages of training. Graph shows total number of days to reach criterion. $(B)$ Total number of days to reach criterion across each of four training stages. (C) Performance $( \pm$ SEM) on odor pairs presented during probe testing. (SHAM) Sham-operated group; (MPFCX) medial prefrontal lesion group; $\left(^{*}\right) P<0.05$.

Animals with damage to the medial prefrontal cortex demonstrate impaired performance on BD (transitive) probe tests and normal performance on AE (nontransitive) probe tests

Mice with damage to the prefrontal cortex were severely impaired in performance on the $\mathrm{BD}$ transitive pair during probe testing. In contrast, these animals performed as well as controls on the AE nontransitive pair. Thus, the animals with prefrontal lesions were impaired selectively in their ability to infer the relationship between previously learned elements that were indirectly related via other elements and were not impaired at making a novel choice between familiar items with consistent reward associations. The initial impairment in acquisition of the odor pairs may have resulted in a less well integrated relational network such that the animals with prefrontal damage were unable to identify indirect relations between the items when challenged with the presentation of the transitive probe test. Despite the fact that animals with prefrontal lesions required twice the number of trials to learn the training

2004). For example, the Wisconsin Card Sort test assesses the ability to exhibit flexible behavior when task contingencies are unexpectedly changed. Patients with frontal lobe damage display a high rate of perseveration, remaining fixed on the previous rule, which is likely due to an inability to inhibit previously reinforced responses (Milner 1963). Damage to the dorsolateral prefrontal cortex in monkeys produces a similar deficit (Dias et al. 1996).

Additionally, tasks that evaluate the ability to establish and shift specific attentional sets have been developed to elucidate the contributions of different regions of the prefrontal cortex (Owen et al. 1991). The set-shifting task evaluates the ability to learn a series of discriminations with various cues ("dimensions"), one of which designates the rewarded item in the pair. Once the rule for discriminations has been acquired, the cue or "dimension" that had previously predicted reward is switched such that a different cue must be followed in order to acquire this new rule (Stefani et al. 2003). Birrell and Brown (2000) demonstrated that the medial prefrontal cortex is critical in learning to attend to a new dimension in rats. Recently, these results were extended to mice as well (Bissonette et al. 2008). This evidence suggests that damage to the medial prefrontal cortex may interfere with the ability to inhibit responding to previously reinforced odors during training (e.g., A is rewarded in the context of $\mathrm{A}$ and $\mathrm{B}$, but $\mathrm{B}$ is rewarded in the context of $\mathrm{B}$ and $\mathrm{C}$ ); therefore, animals are unable to flexibly switch to the correct choice in a situation in which reward contingencies are unexpectedly changed.
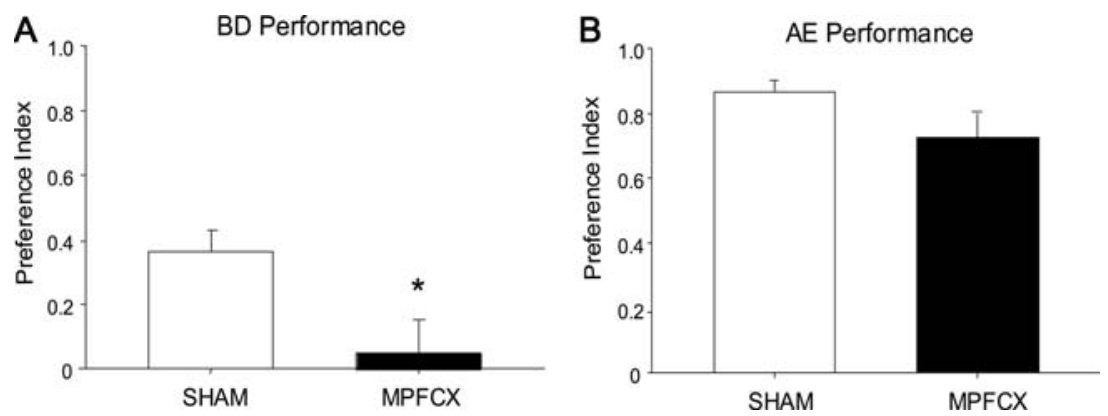

Figure 4. Probe pair performance. $(A)$ Preference index for the transitive probe $B$ vs. D. (B) Preference index for the nontransitive probe A vs. E. (SHAM) Sham-operated group; (MPFCX) medial prefrontal lesion group; $\left(^{*}\right) P<0.05$. 
were trained to learn shape discriminations in an 11-item series, resulted in significant activation of the dorsolateral prefrontal cortex. This activation was specific to the transitive judgments, since performance on a control task in which participants were asked to make a height comparison between different shapes resulted in activation of only the middle frontal gyrus and precentral gyrus. These results suggest that, in addition to supporting the integration of relations between items, the prefrontal cortex may play a dynamic role in the transitive judgment itself.

Neuropsychological studies that have employed the Raven's Progressive Matrices test, a standard neuropsychological task used to assess inductive reasoning, have consistently found that patients with damage to the frontal lobes have difficulty with the planning and manipulation of information required for relational reasoning. Waltz et al. (1999) demonstrated that patients in the early stages of fronto-temporal dementia, when damage is mostly restricted to the prefrontal cortex, were unable to make judgments that required relational processing, while their episodic memory capabilities were superior to that of patients with temporal lobe damage. Additionally, the Tower of London task, a test of motor planning in which the minimum number of moves used to complete the task reflects the most efficient strategy, is also dependent on the functional integrity of the frontal lobes (Dagher et al. 1999). Studies using these tasks have also found that the degree of impairment scales with the level of complexity or the number of multiple relations to be integrated in the task. In addition, the degree of prefrontal activation scales with the amount of relational complexity associated with the task (Christoff et al. 2001; Kroger et al. 2002). Thus, taken together, these studies suggest that the cognitive mechanisms that support relational reasoning rely critically on the functional integrity of the prefrontal cortex (Goel 2007).

\section{Impaired relational memory due to dysfunction of the hippocampal-prefrontal pathway}

Impaired performance on the $\mathrm{BD}$ transitive pair by mice with damage to the prefrontal cortex may be due to dysfunctional circuitry between the hippocampus and the prefrontal cortex. Recent studies have demonstrated that the hippocampus itself is initially critical to the transitive judgment (Van der Jeugd et al. 2009; DeVito et al. 2010). DeVito et al. (2010) found that damage to the hippocampus produced even after the animals had demonstrated successful transitive performance still impairs the ability to perform transitive inference; however, an additional 1 -wk test revealed a partial recovery in relational memory abilities. In contrast to these results, Van der Jeugd et al. (2009) demonstrated that damage to the hippocampus prior to learning, and not after the pairs had been learned, resulted in a deficit in transitive inference judgments. It is important to note that the Van der Jeugd et al. (2009) study targeted the dorsal CA1 region, as compared to the full extent of the hippocampus, which was ablated in the DeVito et al. (2010) study. Regardless of the differences in patterns of results, both studies suggest that other cortical regions, perhaps the medial prefrontal cortex or entorhinal cortex, may be recruited in order to compensate for the loss of hippocampal input. Additionally, the medial prefrontal cortex may support learning the training pairs in transitive inference rather than the hippocampus, since animals with hippocampal lesions are not impaired at learning the odor pairs and perform comparably as their sham-operated controls (Bunsey and Eichenbaum 1996).

There are also important differences between the effects of damage to the prefrontal cortex as compared to the hippocampus on performance with the odor pairs. DeVito et al. (2010) found that animals with hippocampal lesions and sham-operated controls exhibited the same pattern of performance on the odor pairs, such that performance on the context-dependent $\mathrm{BC}$ and $\mathrm{CD}$ discriminations was lower than performance on the $\mathrm{AB}$ and DE pairs; however, the animals with hippocampal damage exhibited higher levels of performance on the end-anchored (AB and $\mathrm{DE}$ ) discriminations as compared to controls. In this study, both sham-operated animals and animals with prefrontal damage exhibited very different patterns of performance. Sham-operated mice exhibited the lowest performance on the BC discrimination as compared to all other pairs, whereas prefrontal lesion animals exhibited the highest performance on the $\mathrm{BC}$ pair. One possible explanation for this pattern of results is that animals with hippocampal damage are more driven by the reinforcement histories of the item associations, whereas animals with prefrontal damage have initial difficulty learning the pairs, resulting in a disrupted network of related information. Although animals in the DeVito et al. (2010) study received damage to the hippocampus after initially learning the pairs, these data indicate that both the prefrontal cortex and the hippocampus play distinct roles in learning and integrating the pairs.

There is considerable evidence suggesting a dynamic interaction between the hippocampus and prefrontal cortex in support of relational memory. For example, the social transmission of food preference task is a test of nonspatial associative memory. This paradigm takes advantage of an animal's neophobia towards novel food items, as this reflects an evolutionary mechanism protecting the animal from ingesting a poisonous food. However, if an animal smells the scent of a novel food on the breath of a conspecific, it will then choose to eat this food as it realizes that it must be safe. Previous research has demonstrated that this memory is dependent on the hippocampus, as well as the prefrontal cortex (Alvarez et al. 2001; Boix-Trelis et al. 2007). However, the hippocampus and prefrontal cortex play different roles, such that both the prefrontal cortex and hippocampus are required for long-term expression of the memory, but only the prefrontal cortex is necessary for the initial acquisition of the memory (Carballo-Marquez et al. 2009). Additionally, the prefrontal cortex also plays a more important role when the test involves greater memory demands, emphasizing the importance of the prefrontal cortex in directing responses when multiple strategies can be employed in order to guide behavioral responses (Winocur and Moscovitch 1999).

Recent imaging studies exploring the time course of activation of the hippocampus and prefrontal cortex during transitive inference support this view. Opstal et al. (2008) and Wendelken and Bunge (2009) found that, in contrast to the hippocampus, the prefrontal cortex exhibited stronger activation on trials that demanded relational integration of multiple items. The hippocampus maintained a similar level of activation across the trials. These results are consistent with the role of the hippocampus in encoding relations between multiple items in support of relational memory and suggest that the prefrontal cortex is involved more specifically in the active integration of multiple relations in working memory. Wendelken and Bunge (2009) also found that there was a strong correlation between the two structures during transitive inference performance, suggesting that an active network is required in order to process multiple relations in memory. Thus, the prefrontal cortex is likely working to organize and establish efficient behavioral strategies that can then be used to integrate multiple associations to support the relational memory processes carried out by the hippocampus.

Additional support for this view comes from recent studies showing that hippocampal and prefrontal damage both result in deficits in recognition memory supported by episodic recollection and not familiarity, as revealed by signal detection analyses (Fortin et al. 2004; Farovik et al. 2008). Whereas damage to both 
areas resulted in an overall deficit, the pattern of impairment was complementary. Thus, hippocampal damage resulted in forgetting previously experienced items, whereas damage to the prefrontal cortex resulted in false memories for items not presented, consistent with a deficit in remembering the list on which previous items were experienced. The combination of results suggest that the hippocampus is critical to encoding and retrieving memories of the elements and the context in which they were experienced, whereas the prefrontal cortex monitors the match between retrieved items and contexts.

Our results suggest that the prefrontal cortex is necessary for transitive inference performance and is involved in relational memory expression. However, the impairment in $\mathrm{BD}$ performance may result either from a lack of proper integration of the odor pairs into memory or an impairment that is specific to the linking of those memories necessary to solve the transitive judgment. Future studies might investigate the effect of inactivation, or a post-training lesion method, to assess the role of the medial prefrontal cortex in transitive inference performance. Alternatively, an anatomical disconnection investigating how damage to the hippocampo-prefrontal pathway affects transitive inference might aid our understanding of the involvement of these two systems in relational memory. The results presented here, in addition to a growing number of studies that aim to elucidate the specific memory processes supported by the prefrontal cortex in the rodent, add to the breadth of behavioral tasks demonstrating functional similarities between human, primate, and rodent prefrontal cortex. Furthermore, the findings on transitive inference add to the number of available tools we can use to identify the specific impairments in animal models of cognitive disorders, as well as the contributions of the hippocampus and prefrontal cortex in supporting essential features of episodic memory.

\section{Materials and Methods}

\section{Subjects}

Male C57 Bl6 mice were purchased from the Charles River Laboratory (Wilmington, MA). All animals were maintained on a reverse 12-h light/dark cycle [09:00 off; 21:00 on]. Animals were given ad libitum access to food and water, unless otherwise specified in the behavioral methods. Nineteen animals were used in this study: 10 animals received lesions of the medial prefrontal cortex, and nine served as sham-operated controls. The IACUC of Boston University approved the treatment and use of the animals in these experiments.

\section{Surgery}

Bilateral lesions of the medial prefrontal cortex were made using ibotenic acid (Tocris Cookson, $0.06 \mathrm{M}$ ) delivered via a microinfusion pump connected to a $10-\mu \mathrm{L}$ Hamilton syringe attached to a pulled microglass pipette tip; sterile saline was used for sham operations. Animals were anesthetized with a ketamine/xylazine cocktail $(0.01 \mathrm{~mL} / \mathrm{g})$, and diazepam $(0.02 \mathrm{~mL})$ was administered preoperatively in order to prevent seizures. After the animal had been placed into a stereotaxic head frame, the skull was exposed and the coordinates of bregma were measured (the medial-lateral values were taken at the level of the mid-sagittal sinus, and not at bregma). The skull overlying the two coordinates was drilled, and dura was removed. The syringe was lowered to the injection site, and the drug was infused over a 5-min period. The needle was left in place for another $5 \mathrm{~min}$ before being slowly withdrawn. The infralimbic and prelimbic cortices were targeted at two stereotaxic coordinates: AP $-2.1, \mathrm{ML} \pm 0.25, \mathrm{DV}-2.3 ; 150 \mathrm{~nL}$ was infused into both sites.

After all infusions, the animal was sutured and given $0.4 \mathrm{~mL}$ of Lactated Ringer's solution and a hotwater bottle in order to hydrate and return body temperature to normal. After surgery, the animal received Children's Tylenol in its water and was provided with soft food and Nutrical. Each animal was allowed 2 wk to recover before behavioral testing.

\section{Odor pair training}

The hierarchical series variant of the transitive inference task was adapted for mice from Dusek and Eichenbaum (1997). Animals were placed on food restriction and maintained at $85 \%$ of free feeding weight. Over a 3-day period animals were allowed to dig for chocolate sprinkle rewards buried in sand that filled small plastic cups. Once they were reliably digging, subjects were given a simple olfactory discrimination of 10 trials across $2 \mathrm{~d}$ in order to teach them to dig in a cup guided by the odor of the sand. All stimuli were composed at $1 \%$ concentration of odorant by weight in sand.

Following the preliminary discrimination problem, animals were trained on a series of overlapping odor pairs (A+vs. B-, $\mathrm{B}+$ vs. $\mathrm{C}-, \mathrm{C}+$ vs. $\mathrm{D}-$, and $\mathrm{D}+$ vs. $\mathrm{E}-$; where $\mathrm{A}$ is paprika [CVS Brand], B is coffee [Folger's], C is basil [McCormick], D is cumin [McCormick], and $\mathrm{E}$ is cocoa [Hershey's]; + and - refer to rewarded and nonrewarded odors, respectively; Fig. 1). Training consisted of two 8-trial sessions per day (separated by a minimum of $30 \mathrm{~min}$ ) across four training stages that began with large blocks of trials of the same discrimination and then involved progressively greater intermingling of the presentations of the different odor pairs, as outlined in the table in Figure 1A. A choice was defined by a significant displacement of the sand by the mouse's paw. After the first training stage, a reward was no longer buried in the sand, but instead a sprinkle was dropped onto the cup if the animal chose correctly. This helped prevent the animal's digging behavior from extinguishing during probe trials in which the cups were not baited. Animals were trained to reach a criterion of $75 \%$ accuracy on each pair across two consecutive days (i.e., 6 out of 8 trials on each of the four odor pairs) at each stage of training.

\section{Probe tests}

The day after reaching criterion on the last training stage, animals were given probe tests for transitive (B vs. D) and nontransitive (A vs. E) pairs, novel choices between items that had not previously been presented together (Fig. 1B). Four BD and four AE probe tests were intermixed with presentations of the odor pairs over a 2-d period, given at trials $3,6,11$, and 14 within the 16 trials. A correct judgment (choosing B over D) on the BD probe required that animals had linked the odor pairs so that they could make the inference across the missing overlapping element $\mathrm{C}$. In contrast, a correct judgment of A over E could be made without reference to the structure of the odor pairs because odor A was always rewarded and odor $\mathrm{E}$ was never rewarded; the $\mathrm{AE}$ pair served as a control for the presentation of novel pairs. Neither cup was baited during probe trials, and the amount of time the animals spent digging in each cup was used as the measure of transitive choice. These digging times were used to calculate a preference index (PI) (DeVito et al. 2010). For the B vs. D test, PI $=(B-D) /$ $(\mathrm{B}+\mathrm{D})$; for A vs. $\mathrm{E}, \mathrm{PI}=(\mathrm{A}-\mathrm{E}) /(\mathrm{A}+\mathrm{E})$.

\section{Histology}

After behavioral testing, all animals were given an overdose of sodium pentobarbital and perfused transcardially with $4 \%$ formalin. The brains were removed and post-fixed for an hour in formalin, and then cryoprotected in $30 \%$ sucrose solution (in $7.4 \mathrm{pH}$ PBS). Coronal sections were cut $(40 \mu \mathrm{m})$ using a freezing microtome. Every section was mounted on gelatin-coated slides and dried overnight. Slides were soaked in xylenes and then run through a series of ethanol dehydrations, stained with cresyl violet, and then rehydrated. The extent of the lesion was determined using a light microscope to study the stained sections.

Three representative sections along the anterior-posterior axis of the medial prefrontal cortex (AP: $-2.58,-2.1,-1.7$ ) were selected from the mouse brain atlas in order to determine tissue damage (Franklin and Paxinos 1997). Canvas 5.0 (Deneba 
Software, ACD Systems International Inc.) was used to calculate the area of the infralimbic and prelimbic cortices in each section. For each animal, the three sections most closely corresponding to the representative sections in the brain atlas were used. Percent damage was calculated as the amount of total damage divided by the total area of the prefrontal cortex in that section $\times 100$; the average of those three values represented the lesion extent. Additional sections were studied under the light microscope in order to determine if any incidental damage occurred outside the targeted regions, and these are reported in the Results section.

\section{Acknowledgments}

We thank Anja Farovik for her thoughtful comments on the manuscript. This work was funded by the Conte Center for Schizophrenia Research (NIH MH60450). The results of this study were presented at the Society for Neuroscience 2009 meeting.

\section{References}

Acuna BD, Eliassen JC, Donoghue JP, Sanes JS. 2002. Frontal and parietal activation during transitive inference in humans. Cereb Cortex 12: 1312-1321.

Alvarez P, Lipton PA, Melrose R, Eichenbaum H. 2001. Differential effects of damage within the hippocampal region on memory for a natural, nonspatial odor-odor association. Learn Mem 8: 79-86.

Birrell JM, Brown VJ. 2000. Medial frontal cortex mediates perceptual attentional set shifting in the rat. J Neurosci 20: 4320-4324.

Bissonette GB, Martins GJ, Franz TM, Harper ES, Schoenbaum G, Powell EM. 2008. Double dissociation of the effects of medial and orbital prefrontal cortical lesions on attentional and affective shifts in mice. J Neurosci 44: $11124-11130$.

Boix-Trelis N, Vale-Martinez A, Guillazo-Blanch G, Marti-Nicolovius M. 2007. Muscarinic cholinergic receptor blockade in the rat prelimbic cortex impairs the social transmission of food preference. Neurobiol Learn Mem 87: 659-668.

Brown VJ, Bowman EM. 2002. Rodent models of prefrontal cortical function. Trends Neurosci 25: 340-343.

Buckmaster CA, Eichenbaum H, Amaral DG, Suzuki WA, Rapp PR. 2004. Entorhinal cortex lesions disrupt the relational organization of memory in monkeys. J Neurosci 44: 9811-9825.

Buckner RL, Kelley WM, Petersen SE. 1999. Frontal cortex contributes to human memory formation. Nature 2: 311-314.

Bunsey M, Eichenbaum H. 1996. Conservation of the hippocampal memory function in rats and humans. Nature 379: 255-257.

Carballo-Marquez A, Vale-Martinez A, Guillazo-Blanch G, Marti-Nicolovius M. 2009. Muscarinic receptor blockade in ventral hippocampus and prelimbic cortex impairs memory for socially transmitted food preference. Hippocampus 19: 446-455.

Carpenter PA, Just MA, Reichle ED. 2000. Working memory and executive function: Evidence from neuroimaging. Curr Opin Neurobiol 10: 195-199.

Christoff K, Prabhakaran V, Dorfman J, Zhao Z, Kroger JK, Holyoak KJ, Gabrieli JD. 2001. Rostrolateral prefrontal cortex involvement in relational integration during reasoning. NeuroImage 14: 1136-1149.

Dagher A, Owen AM, Boecker H, Brooks DJ. 1999. Mapping the network for planning: A correlational PET activation study with the Tower of London task. Brain 122: 1973-1987.

Dalley JW, Cardinal RN, Robbins TW. 2004. Prefrontal executive and cognitive functions in rodents: Neural and neurochemical substrates. Neurosci Biobehav Rev 28: 771-784.

DeVito LM, Kanter BR, Eichenbaum H. 2010. The hippocampus contributes to memory expression during transitive inference in mice. Hippocampus 20: 208-217.

Dias R, Robbins TW, Roberts AC. 1996. Dissociation in prefrontal cortex of affective and attentional shifts. Nature 380: 69-72.

Dusek JA, Eichenbaum H. 1997. The hippocampus and memory for orderly stimulus relations. Proc Natl Acad Sci 94: 7109-7114.

Eichenbaum H. 2004. Hippocampus: Cognitive processes and neural representations that underlie declarative memory. Neuron 44: $109-120$.
Farovik A, DuPont L, Arce M, Eichenbaum H. 2008. Medial prefrontal cortex supports recollection, but not familiarity, in the rat. J Neurosci 28: $13428-13434$

Feredoes E, Tononi G, Postle BR. 2006. Direct evidence for a prefrontal contribution to the control of proactive interference in verbal working memory. Proc Natl Acad Sci 103: 19530-19534.

Fortin N, Wright S, Eichenbaum H. 2004. Recollection-like memory retrieval in rats is dependent on the hippocampus. Nature 431: $188-191$.

Franklin BJ, Paxinos G. 1997. The mouse brain in stereotaxic coordinates. Academic Press, San Diego, CA.

Goel V. 2007. Anatomy of deductive reasoning. Trends Cog Sci 11: $435-441$.

Goel V, Dolan RJ. 2001. Functional neuroanatomy of three-term relational reasoning. Neuropsychologia 39: 901-909.

Heckers S, Zalesak M, Weiss A, Ditman T, Titone D. 2004. Hippocampal activation during transitive inference in humans. Hippocampus 14: $153-162$.

Krawczyk DC, Morrison RG, Viskontas I, Holyoak KJ, Chow TW, Mendez MF, Miller BL, Knowlton BJ. 2008. Distraction during relation reasoning: The role of the prefrontal cortex in interference control. Neuropsychologia 46: 2020-2032.

Kroger JK, Sab FW, Fales CL, Bookheimer SY, Cohen MS, Holyoak KJ. 2002. Recruitment of anterior dorsolateral prefrontal cortex in human reasoning: A parametric study of relational complexity. Cereb Cortex 12: $477-485$

Laroche S, Davis S, Jay TM. 2000. Plasticity and hippocampal to prefrontal cortex synapses: Dual roles in working memory and consolidation. Hippocampus 10: $438-446$.

Milner B. 1963. Effects of different brain lesions on card sorting. Arch Neurol 9: $90-100$.

Ongur D, Price J. 2000. The organization of networks within the orbital and medial prefrontal cortex of rats, monkeys, and humans. Cereb Cortex 10: $206-219$

Opstal FV, Verguts T, Orban GA, Fias W. 2008. A hippocampal-parietal network for learning an ordered sequence. NeuroImage 40: $333-341$

Owen AW, Roberts AC, Polkey CE, Sahakian BJ, Robbins TW. 1991. Extra-dimensional vs. intra-dimensional set shifting performance following frontal lobe excisions, temporal lobe excisions or amygdalo-hippocampectomy in man. Neuropsychologia 29: 993-1006.

Petrides M. 2000. The role of the mid-dorsolateral prefrontal cortex in working memory. Exp Brain Res 133: 44-54.

Rezai K, Andreasen NC, Alliger R, Cohen G, Swayze V, O'Leary DS. 1993. The neuropsychology of the prefrontal cortex. Arch Neurol 50: 636-642.

Rich EL, Shapiro M. 2009. Rat prefrontal cortical neurons selectively code strategy switches. J Neurosci 29: 7208-7219.

Simons JS, Spiers HJ. 2003. Prefrontal and medial temporal lobe interactions in long-term memory. Nat Rev Neurosci 4: 637-648.

Smith EE, Jonides J. 1999. Storage and executive processes in the frontal lobes. Science 283: 1657-1661.

Stefani M, Groth K, Moghaddam B. 2003. Glutamate receptors in the rat medial prefrontal cortex regulate set-shifting ability. Behav Neurosci 117: $728-737$

Thierry A, Gioanni Y, Degenetais E, Glowinski J. 2000. Hippocampo-prefrontal cortex pathway: Anatomical and electrophysiological characteristics. Hippocampus 10: 411-419.

Van der Jeugd A, Goddyn H, Laeremans A, Arckens L, D'Hooge R, Verguts T. 2009. Hippocampal involvement in the acquisition of relational associations, but not in the expression of a transitive inference task in mice. Behav Neurosci 123: 109-114.

Verwer RW, Meijer RJ, Van Uum HF, Witter MP. 1997. Collateral projections from the rat hippocampal formation to the lateral and medial prefrontal cortex. Hippocampus 7: 397-402.

Waltz JA, Knowlton BJ, Holyoak KJ, Boone KB, Mishkin FS, Santos MD, Thomas CR, Miller BL. 1999. A system for relational reasoning in human prefrontal cortex. Psychol Sci 10: 119-125.

Wendelken C, Bunge SA. 2009. Transitive inference: Distinct contributions of rostrolateral prefrontal cortex and the hippocampus. J Cogn Neurosci doi: 10.1162/jocn.2009.21226.

Winocur G, Moscovitch M. 1999. Anterograde and retrograde amnesia after lesions to frontal cortex in rats. J Neurosci 19: 9611-9617.

Zalesak M, Heckers S. 2009. The role of the hippocampus in transitive inference. Psychiatry Res 172: 24-30.

Received November 6, 2009; accepted in revised form January 10, 2010. 


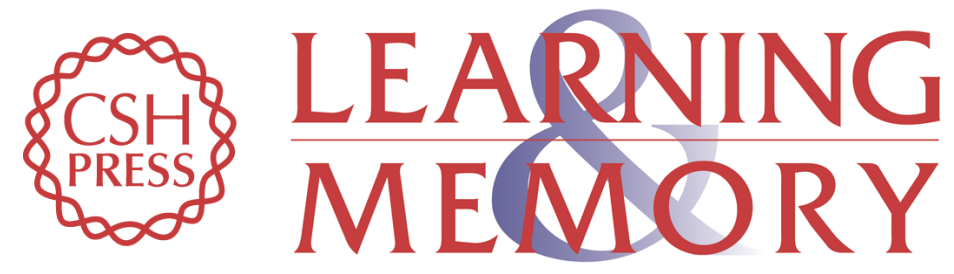

\section{Prefrontal cortex: Role in acquisition of overlapping associations and transitive inference}

Loren M. DeVito, Christine Lykken, Benjamin R. Kanter, et al.

Learn. Mem. 2010, 17:

Access the most recent version at doi:10.1101//m.1685710

References This article cites 43 articles, 8 of which can be accessed free at:

http://learnmem.cshlp.org/content/17/3/161.full.html\#ref-list-1

License

Email Alerting Receive free email alerts when new articles cite this article - sign up in the box at the Service top right corner of the article or click here. 